\title{
Preliminary Program for Mars Samples Analysis
}

\author{
Marie-Christine Maurel \\ Evolutionary Biochemistry and Molecular Adaptation, Jacques-Monod \\ Institute, University Paris VI, Tour 43, 2 place Jussieu, 75251 Paris, \\ France \\ Jean-Louis Counil \\ C.N.E.S. et C.S.E.E.M, Centre de Toulouse, 18 ave. Edouard Belin \\ 31401 Toulouse cedex 4, France
}

\begin{abstract}
The main goals of the French Mars exploration program are the development of a netlander for geophysical studies. This project is conducted in the framework of a consortium of European partners (Finland, Germany and Belgium), and an orbiter vehicle of the future Mars sample return mission (MSR) is being developed in cooperation with NASA. Also, we discuss the preparatory program for volatile and rare gas analysis, sample pollution, site selection and Astrobiology.
\end{abstract}

\section{Introduction}

The main goals of the French Mars exploration program named PREMIER (Programme de Retour d'Echantillons Martiens et d'Installation d'Experiences en Réseau) are the development, deployment and operation of a network of four small Mars ground stations for performing geophysical measurements. This netlander project is conducted in the framework of a consortium of European partners (Finland, Germany and Belgium). The development, deployment and operation of the orbiter vehicle of the future Mars sample return mission (MSR) are also performed in cooperation with NASA.

Its additional elements are: instrument contributions to ESAs Mars Express orbiter mission and payload contributions to the orbiters, landers and rovers to be launched to Mars in 2007 and beyond.

This program is designed to ensure complementarity among three poles: global investigations from orbit, landed science with both network science (NetLanders) and in-situ investigations, and sample return.

\section{Means and Objectives}

In 1999, a French committee, CSEEM (Scientific committee for the study of Mars samples) was created for national and international purposes. In 1999 this committee, mandated to coordinate the national endeavour, released an $\mathrm{AO}$ (Announcement of Opportunity) aimed at implementing a national preparatory program for Mars sample analysis. More than 40 proposals were submitted 
involving more than 450 scientists from around 60 French labs. Most of the proposals are interdisciplinary and were jointly submitted by planetologists, mineralogists, geochemists, astrobiologists and biologists. The first stage of this preparatory program is continuing and will last until mid-2003.

\section{Preparatory Program}

Amongst the priorities of the preparatory program are the development of dedicated instrumentation, the capability of analysing samples as small as possible, and integrated measurements. The measurements will involve rock-macromolecule interactions, behavior of bacteria under Martian conditions, sample transportation under quarantine conditions, and others.

\section{Volatile and rare gas analysis:}

The analysis of rare gas and volatiles is essential to understand the early evolution of Mars and the formation and evolution of its reservoirs (core, mantle, and atmosphere). The analysis of Mars samples will require working on quantities as small as nanomoles and the development of new analysis lines. In preparation for analysis of the Martian samples, studies of the solar wind and samples of asteroids and comets are planned (missions Genesis, MUSEC, Stardust).

\section{Sample pollution:}

To guarantee the quality of the analysis, keeping the sample pristine is a key task. In particular, the tools used to core and bring back samples should not pollute the samples. Metals such as hafnium, lead and tungsten that are involved in isotopic analysis should be avoided.

\section{Site selection:}

Choosing the right place to sample is essential. The habitable zone for life on Mars must be defined in terms of the chemical and physical conditions that are expected to be required for life. The prerequisite is to define sites according to water localization, strong UV irradiation and the presence of oxidants on the surface.

\section{Astrobiology:}

Biochemistry requires water and carbon-based chemistry. Life as we know it is built from simple organic molecules, the building blocks for large molecules. Amino acids, nucleic acid bases and sugars are the biological monomers of macromolecules. It seems logical that the evolution of any organic-based life form would result in the construction of molecules as repeating structures of simple subunits.

The sources of organic molecules from meteorites and comets would be the same on Mars as on Earth and especially on the early Earth (Fig. 1). In the 


\section{Sources of organic molecules on Mars}

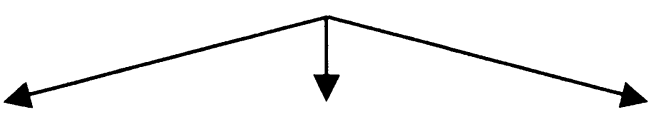

- Biotic source - Meteoritic source

- extant life

- extinct life
- carbonaceous chondrites

- micrometeorites

- planetary meteorites (in less extent)
- Prebiotic chemistry

- subsurface chemistry

- chemistry in a primitive environment

Figure 1. Sources of organic molecules on Mars

expectation that conditions were oxidizing (Bruston et al. 1999), we have to be aware of the necessity to look for traces in the subsoil.

Mars exploration and samples analysis are of fundamental interest for scientists looking for appearance of life on Earth and elsewhere, for those interested in the definition of life and the processes of development of living beings within ecosystems, and for those concerned with molecular mechanisms within the living cell, and processes of adaptation and evolution (Maurel \& Zaccai 2001). Numerous fields play a role: genomics, virology, microbiology, biochemistry, molecular biology, biophysics, and studies of non-conventional organisms. This list is far from exhaustive. For instance, combinatorial methods now permit the investigation of potential diversity of new macromolecules and the re-emergence of ancient ones which have diverged or naturally disappeared (Maurel \& Décout 1999; Benner et al. 2000).

Detection of life and biological tests for possible pathogenicity will be performed during a quarantine phase. Two main lines have priority: definition of a quarantine protocol including characterization analysis, detection and determination of hazards; and perfecting level 4 laboratory methods. After quarantine, microbiologists, virologists and biologists who are experts in pathogen agents (including non-conventional ones) would be involved in confinement and conservation of samples, in the short or long term. In France, a level 4 laboratory (P4) in Lyon, (see http://www.fond-merieux.org), well-equipped with up-to-date facilities, and a confinement place to receive Mars samples, is available.

Several teams of complementary laboratories in France are working together to develop expertise in the identification and reactivity of bio-organic molecules from the Martian samples. The broad outline of this program is to optimize the methodologies, to experiment under conditions mimicking those that are characteristic of Mars (irradiation, temperature, pressure, hydrous potential, salts etc.), and to study micro-organisms adapted to extreme terrestrial environments in order to understand their biochemical machinery. In order to prepare for a search for macromolecular signatures on Mars, it is essential to study the ef- 
fects of the environment and in particular of salts on the structure, stability, interactions and dynamics of molecules from these extremes (Tehei et al. 2002).

\section{References}

Benner, S. A., Devine, K. G., Matveeva, L. N., \& Powell, D. H. 2000, PNAS, 97,2425

Bruston, F., Vergne, J., Grajcar, L., Drahi, B., Calvayrac, R., Baron, M-H., \& Maurel, M. C. 1999, Biochem. Biophys. Res. Commun. 263, 672

Maurel M-C. \& Décout, J-L. 1999, Tetrahedron, 55, 3141

Maurel, M-C., \& Zaccai, G. 2001, BioEssays, 23, 977

Tehei, M., Franzetti, B., Maurel, M. C., Vergne, J., Hountondji, C., \& Zaccai, G. 2002, Extremophiles in press

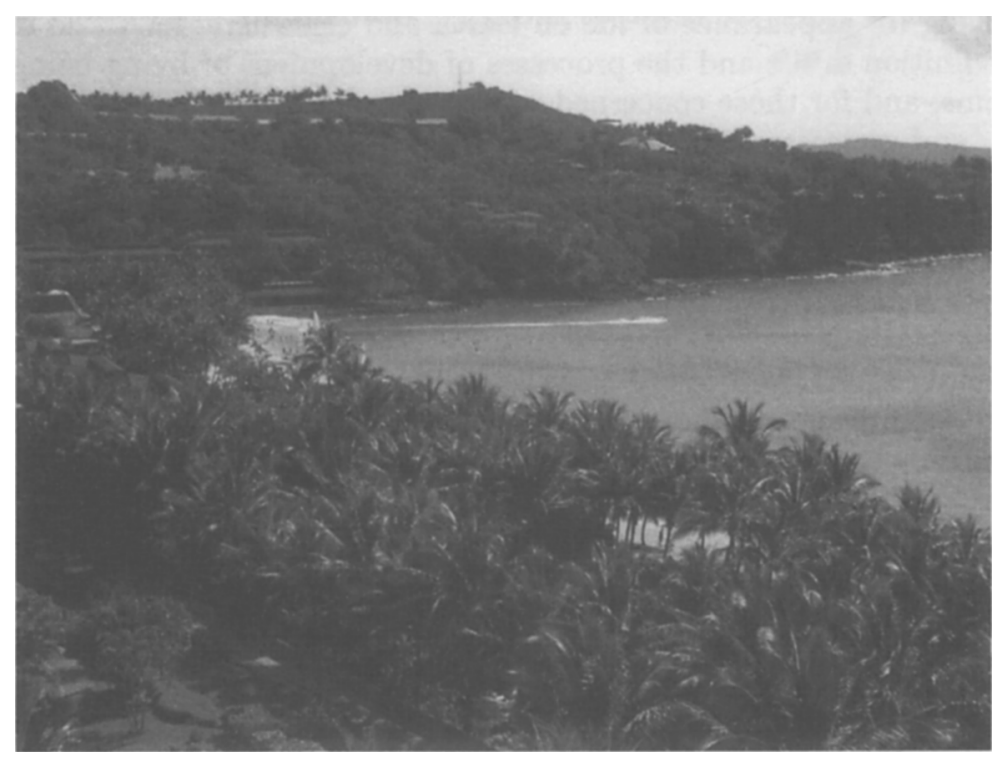

(photo: Stephen Mojzsis) 